\title{
Computational prediction of miRNAs and their targets in Phaseolus vulgaris using simple sequence repeat signatures
}

\author{
Chandran Nithin ${ }^{1 \dagger}$, Nisha Patwa ${ }^{2 \dagger}$, Amal Thomas $^{1 \dagger}$, Ranjit Prasad Bahadur ${ }^{1 *}$ and Jolly Basak ${ }^{2 *}$
}

\begin{abstract}
Background: MicroRNAs (miRNAs) are endogenous, noncoding, short RNAs directly involved in regulating gene expression at the post-transcriptional level. In spite of immense importance, limited information of $P$. vulgaris miRNAs and their expression patterns prompted us to identify new miRNAs in $P$. vulgaris by computational methods. Besides conventional approaches, we have used the simple sequence repeat (SSR) signatures as one of the prediction parameter. Moreover, for all other parameters including normalized Shannon entropy, normalized base pairing index and normalized base-pair distance, instead of taking a fixed cut-off value, we have used $99 \%$ probability range derived from the available data.

Results: We have identified 208 mature miRNAs in P. vulgaris belonging to 118 families, of which 201 are novel. 97 of the predicted miRNAs in P. vulgaris were validated with the sequencing data obtained from the small RNA sequencing of $P$. vulgaris. Randomly selected predicted miRNAs were also validated using qRT-PCR. A total of 1305 target sequences were identified for 130 predicted miRNAs. Using $80 \%$ sequence identity cut-off, proteins coded by 563 targets were identified. The computational method developed in this study was also validated by predicting 229 miRNAs of $A$. thaliana and 462 miRNAs of G. max, of which 213 for A. thaliana and 397 for G. max are existing in miRBase 20.

Conclusions: There is no universal SSR that is conserved among all precursors of Viridiplantae, but conserved SSR exists within a miRNA family and is used as a signature in our prediction method. Prediction of known miRNAs of A. thaliana and G. max validates the accuracy of our method. Our findings will contribute to the present knowledge of miRNAs and their targets in $P$. vulgaris. This computational method can be applied to any species of Viridiplantae for the successful prediction of miRNAs and their targets.
\end{abstract}

Keywords: miRNA, Phaseolus vulgaris, SSRs, Shannon entropy, MFEI

\section{Background}

MicroRNAs (miRNAs) are small non-coding RNAs [1] with an approximate length of 22 nucleotides originating from long self-complementary precursors [2]. miRNA precursor sequences (pre-miRs) have intrinsic hairpin structure which consists of the entire miRNA sequence on one arm of the hairpin and the miRNA* sequence on the opposite arm. miRNAs regulate a variety of

\footnotetext{
* Correspondence: r.bahadur@hijli.iitkgp.ernet.in; jolly.basak@visva-bharati.ac.in ${ }^{\dagger}$ Equal contributors

${ }^{1}$ Computational Structural Biology Lab, Department of Biotechnology, Indian Institute of Technology Kharagpur, Kharagpur 721302, India

${ }^{2}$ Department of Biotechnology, Visva-Bharati, Santiniketan 731235, India
}

biological processes like development, metabolism, stress response, pathogen defense and maintenance of genome integrity $[3,4]$. Mature miRNA gets incorporated into the RNA-induced silencing complex (RISC) [2], which regulates gene expression either by inhibiting translation or by degrading coding mRNAs by perfect or near-perfect complement with the target mRNAs $[5,6]$. For a given miRNA, the number of target mRNA ranges from one to hundreds [7]. However, in plants, most of the target mRNAs contain a single miRNA-complementary site, and the corresponding miRNAs perfectly complement these sites and cleave the target mRNAs [8].

The first miRNA (lin-4) was identified in Caenorhabditis elegans in 1993 [9]. Since then, hundreds of miRNAs 
have been identified in plants, animals and viruses. In recent years, advancement in technologies such as Bioinformatics and Next-Generation Sequencing (NGS) facilitated the identification of huge number of putative miRNAs in different organisms. However, the process of identifying miRNAs is still a complex and difficult task requiring interdisciplinary strategies, including experimental approaches as well as computational methods. Compared to the experimental approaches, computational predictions have been proved to be fast, affordable, and accurate [10-26]. In the last ten years, different computational strategies have been developed to find new miRNAs, including mining the repository of available Expressed Sequence Tags (ESTs) with known miRNAs, as well as those based on the conserved nature of miRNAs [12-16, 22, 23].

Majority of miRNAs are evolutionarily conserved between different species of the same kingdom and may also exist as orthologs or homologs in other species [27]. Computational prediction of putative miRNAs is often based on their evolutionarily conserved nature. Accordingly, homologs of known miRNAs are searched in the EST databases to identify the putative pre-miRs in other species. Pre-miRs have a specific range of percentage AU content in their sequences as well as Minimal Folding free Energy Index (MFEI) [27]. Studies have also shown that pre-miRs have distinct RNA folding measures such as normalised Shannon entropy (NQ), normalized base-pair distance (ND) and normalized base-pairing propensity $(\mathrm{Npb})$. Thus, AU content and MFEI are also used as parameters for prediction of new miRNAs.

Simple sequence repeats (SSRs) are repeating sequences of one to six nucleotides long [28]. The presence of SSRs in pre-miRs was identified by several studies [29-31], although their precise role in pre-miRs is yet to be elucidated. The SSRs present in pre-miRs in different species did not show noticeable locational preferences and are found anywhere in pre-miRs, suggesting that SSRs are the important component of pre-miRs [32]. In pre-miRs, mononucleotide repeats are the most abundant repeats, followed by di- and tri-nucleotide repeats, while tetra-, penta-, and hexanucleotide repeats rarely occur [32]. Moreover, the number of repeats correlates inversely to the length of the repeats [32]. Absence of long SSRs and low number of repeat types in pre-miRNAs may be attributed to their small size, stability and low mutation rate [32]. Due to these very characteristics, the identification of SSR signatures in pre-miRs is easy and can be used as a parameter in predicting miRNAs. However, SSR signatures have not been used in the computational prediction of new miRNAs. In the present study, we have used SSR signatures as a parameter to predict new miRNAs.

Phaseolus vulgaris, belonging to the Fabaceae family, is a vital leguminous crop in tropical and subtropical areas of Asia, Africa, and Latin America, as well as parts of southern Europe and the USA (FAOSTAT 2009). P. vulgaris is an important food worldwide and a significant source of fibre, proteins and vitamins (FAOSTAT 2009). High protein and carbohydrate content makes it not only important for the human diet, but also suitable as high protein feed and fodder for livestock. $P$. vulgaris is a particular valuable component of low-input farming system of resource-poor farmers (FAOSTAT 2009). This leguminous crop enhances soil fertility through nitrogen fixation [33]. In spite of immense importance, limited information is available about the miRNAs of $P$. vulgaris and their patterns of expression [34-40]. There are only eight reported miRNAs of $P$. vulgaris in the miRBase 20 [41]. In the present study, we have identified new miRNAs in P. vulgaris by computational methods. In addition to the conventional approaches, we have used the conserved SSR signatures as one of the parameters for prediction. Moreover, for all the other parameters, instead of considering a fixed cut-off value, we have used a $99 \%$ probability range derived from the available data. We obtained 208 new miRNAs, of which 201 are novel. Few randomly selected predicted miRNAs were validated using qRT-PCR. Targets for many of the predicted miRNAs were identified. Additionally, we also validated our computational method by predicting known miRNAs in A. thaliana and G. max. Our findings will contribute to the present knowledge of miRNAs and their targets in $P$. vulgaris. The computational method developed in this study is not only restricted to $P$. vulgaris but can be applied to any species of Viridiplantae.

\section{Results}

Analysis of known Viridiplantae pre-miRs

All the known 6088 pre-miRs of Viridiplantae in the miRBase 20 [41] were analysed, and the probability distributions of their AU content, length and MFEI are shown in Fig. 1. The length of pre-miRs varies from 43 to 938 nucleotides, with the mean value of 149 . However, when we consider the $99 \%$ probability range, the length of pre-miRs varies from 55 to 505 nucleotides. Consequently, we set this range as a cut-off value for the prediction of new miRNAs. The percentage of AU content in the pre-miRs ranges from $17 \%$ to $92 \%$. This range becomes $27 \%$ to $77 \%$ when we consider the $99 \%$ probability region, and accordingly it is used as the AU content cut-off range. The MFEI has a mean value of $1.0 \pm 0.28$, however while considering $99 \%$ probability range, it is greater than or equal to 0.41 . Consequently, this value is used as the cut-off for MFEI. The probability distributions for ND, NQ and $\mathrm{Npb}$ are plotted in Fig. 2. Considering the $99 \%$ probability region in the distribution, the values of NQ and ND are less than or equal to 0.45 and 0.15 , respectively, while for $\mathrm{Npb}$ it is 

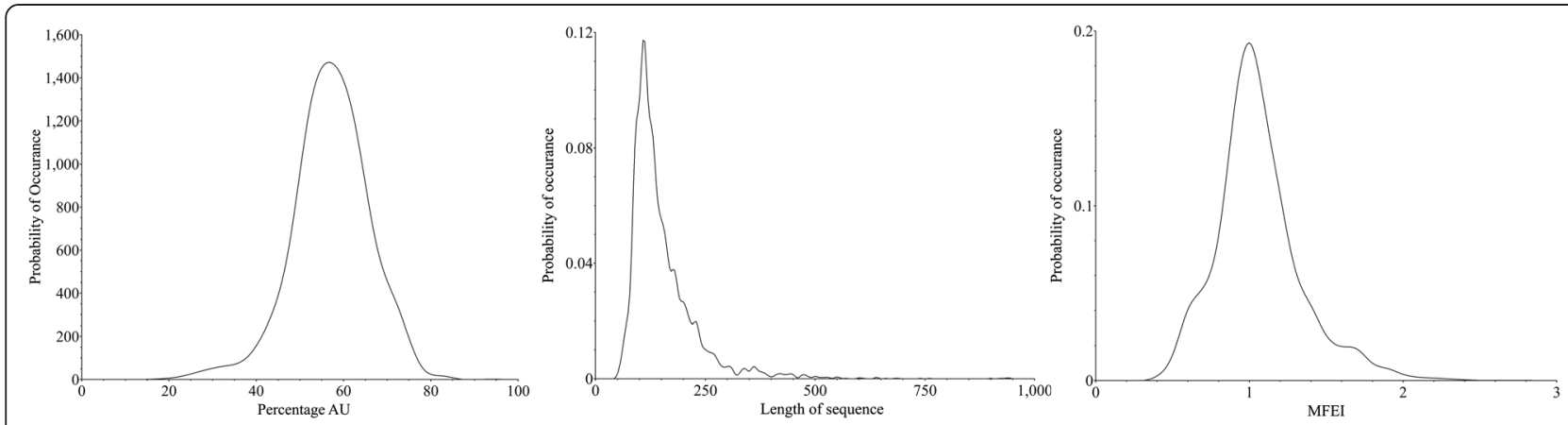

Fig. 1 Probability distributions of percentage AU content, length and MFEl of pre-miRs belonging to Viridiplantae

greater than or equal to 0.25 . These values have been used as the cut-off for these parameters.

\section{Simple Sequence Repeats (SSRs)}

To find the conserved SSR signatures within the premiRs, all the 1892 miRNA families of Viridiplantae were analysed (Additional file 1 Table S1). None of the SSR signatures were found to be conserved in all the families. However, conserved SSR signature(s) was found when a particular family was considered. We find 1427 families with only one pre-miR, and 465 families with two or more pre-miRs. Within these 465 families, only those conserved SSRs that are present in all the members of a particular family were considered. The conserved SSR having the maximum average $\mathrm{R}$ (number of SSR signatures per 100 nucleotides) value was chosen as a SSR signature for a given family. We find that with the window size three, the average $\mathrm{R}$ of a signature SSR is greater than 2.5. With the increase in the window size, the number of miRNA families having a conserved SSR signature with an average $\mathrm{R}$ greater than two becomes limited. Accordingly, the window size three was set to identify the conserved SSR signatures in pre-miRs. For the 1427 families with only one pre-miR, the SSR with the maximum $\mathrm{R}$ was selected as a signature. In single member families, the $\mathrm{R}$ is always greater than 2.5 , which is the minimum average $R$ for the SSR signatures found in the multimember families.

The SSR signatures in different miRNA families of the kingdom Viridiplantae, the family Fabaceae and the species $P$. vulgaris were analysed in Table 1 . It shows that in Viridiplantae, $8.77 \%$ of miRNA families contain the signature AUU, $7.45 \%$ of miRNA families contain the signature AAU and $6.29 \%$ of miRNA families contain the signature UUU. In Fabaceae, $10.71 \%$ of miRNA families contain the signature AUU, $9.70 \%$ of miRNA families contain the signature AAU and $6.87 \%$ of miRNA families contain the signature UUU. In $P$. vulgaris, the signature UUG is present in $15.25 \%$ of miRNA families, while both the signatures AUU and UUU are present in $10.17 \%$ of miRNA families. Significantly, the three most frequently found signatures in each taxonomic category are found in most of the miRNA families. They are the signatures of $23 \%$ miRNA families in Viridiplantae, of $27 \%$ miRNA families in Fabaceae and of $36 \%$ miRNA families in P. vulgaris. The signature CCC is found in only one miRNA family in Viridiplantae, and is absent in all miRNA families in Fabaceae as well as in P. vulgaris.
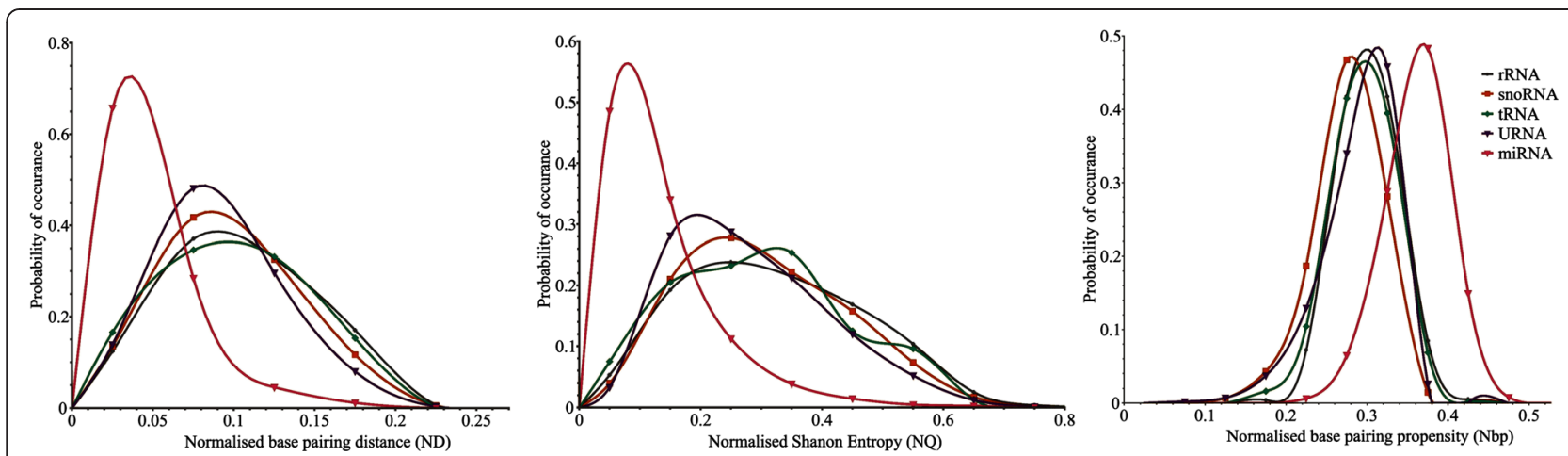

Fig. 2 Probability distributions of normalized base-pair distance (ND). normalized Shannon entropy (NQ) and normalized base pairing propensity $(\mathrm{Npb})$ of pre-miRs belonging to Viridiplantae 
Table 1 Distribution of SSR signatures in various miRNA families of Viridiplantae, Fabaceae and P. vulgaris

\begin{tabular}{|c|c|c|c|c|c|c|c|c|c|c|c|c|c|}
\hline & A & & & U & & & $C$ & & & G & & & \\
\hline & $V^{a}$ & $F^{b}$ & $P^{c}$ & $\mathrm{~V}^{\mathrm{a}}$ & $F^{b}$ & $P^{c}$ & $\mathrm{~V}^{\mathrm{a}}$ & $F^{b}$ & $P^{c}$ & $\mathrm{~V}^{\mathrm{a}}$ & $F^{b}$ & $P^{c}$ & \\
\hline \multirow[t]{4}{*}{$\mathrm{A}$} & 4.92 & 4.44 & 2.54 & 2.96 & 1.41 & 1.69 & 1.32 & 1.82 & 0.85 & 1.48 & 2.02 & 4.24 & ${ }^{A}$ \\
\hline & 7.45 & 9.70 & 5.93 & 8.77 & 10.71 & 10.17 & 0.79 & 1.01 & 0.85 & 0.79 & 0.61 & 0.00 & U \\
\hline & 1.43 & 1.41 & 0.85 & 2.38 & 3.03 & 0.85 & 0.63 & 0.61 & 1.69 & 1.06 & 0.40 & 0.00 & c \\
\hline & 3.07 & 4.04 & 2.54 & 3.91 & 2.83 & 5.08 & 0.37 & 0.20 & 0.00 & 0.69 & 0.20 & 0.00 & G \\
\hline \multirow[t]{4}{*}{ U } & 2.01 & 1.82 & 0.00 & 2.17 & 3.23 & 0.85 & 1.80 & 2.63 & 0.85 & 2.70 & 3.03 & 4.24 & A \\
\hline & 2.59 & 2.83 & 2.54 & 6.29 & 6.87 & 10.17 & 2.17 & 1.82 & 1.69 & 2.48 & 1.62 & 0.00 & U \\
\hline & 0.21 & 0.00 & 0.00 & 2.27 & 3.03 & 5.08 & 0.85 & 0.61 & 0.00 & 1.48 & 0.40 & 1.69 & C \\
\hline & 0.58 & 0.61 & 0.00 & 6.18 & 6.46 & 15.25 & 0.58 & 0.81 & 0.85 & 1.53 & 1.62 & 0.85 & $C$ \\
\hline \multirow[t]{4}{*}{ C } & 1.22 & 1.01 & 3.39 & 0.37 & 0.00 & 0.00 & 0.79 & 0.61 & 0.00 & 0.32 & 0.20 & 0.00 & A \\
\hline & 1.90 & 2.22 & 0.00 & 2.11 & 2.22 & 5.08 & 0.32 & 0.40 & 0.00 & 0.37 & 0.81 & 0.00 & U \\
\hline & 0.26 & 0.20 & 0.00 & 0.79 & 0.20 & 0.00 & 0.05 & 0.00 & 0.00 & 0.69 & 0.00 & 0.85 & C \\
\hline & 0.37 & 0.20 & 0.00 & 0.63 & 0.40 & 0.00 & 0.63 & 0.00 & 0.85 & 0.74 & 0.40 & 0.85 & G \\
\hline \multirow[t]{4}{*}{ G } & 1.48 & 2.42 & 2.54 & 0.32 & 0.00 & 0.00 & 0.69 & 0.40 & 0.00 & 0.79 & 0.81 & 0.00 & A \\
\hline & 1.59 & 1.62 & 2.54 & 0.95 & 1.41 & 0.85 & 0.58 & 0.81 & 0.00 & 0.42 & 0.40 & 0.00 & U \\
\hline & 0.16 & 0.40 & 0.00 & 0.26 & 0.20 & 0.00 & 0.74 & 0.20 & 0.00 & 0.90 & 0.20 & 0.00 & C \\
\hline & 0.58 & 0.20 & 1.69 & 0.16 & 0.00 & 0.00 & 0.69 & 0.20 & 0.00 & 0.21 & 0.00 & 0.00 & G \\
\hline
\end{tabular}

$\mathrm{V}^{\mathrm{a}}$ - The percentage of miRNA families belonging to Viridiplantae with a particular signature SSR. There are 1892 miRNA families to which Viridiplantae miRNAs belong. $F^{b}$ - The percentage of miRNA families belonging to Fabaceae with a particular signature SSR. There are 495 miRNA families to which $P$. vulgaris miRNAs belong. $P^{c}$ - The percentage of miRNA families belonging to $P$. vulgaris with a particular signature SSR. There are 118 miRNA families to which $P$. vulgaris miRNAs belong

In Fabaceae, eight signatures are absent in all miRNA families, while 11 signatures are found only in one miRNA family. In P. vulgaris, 32 out of 64 signatures are absent in all miRNA families. The relative distribution of the SSR signatures in the Viridiplantae, Fabaceae and $P$. vulgaris is shown in Fig. 3.

\section{Prediction of new miRNAs in $P$. vulgaris}

The known Viridiplantae miRNAs from the miRBase 20 were used as query in the BLAST search with the EST and GSS sequences of $P$. vulgaris as subject. From the BLAST results satisfying the conditions mentioned in the 'materials and methods' section, a total of 141,724,357

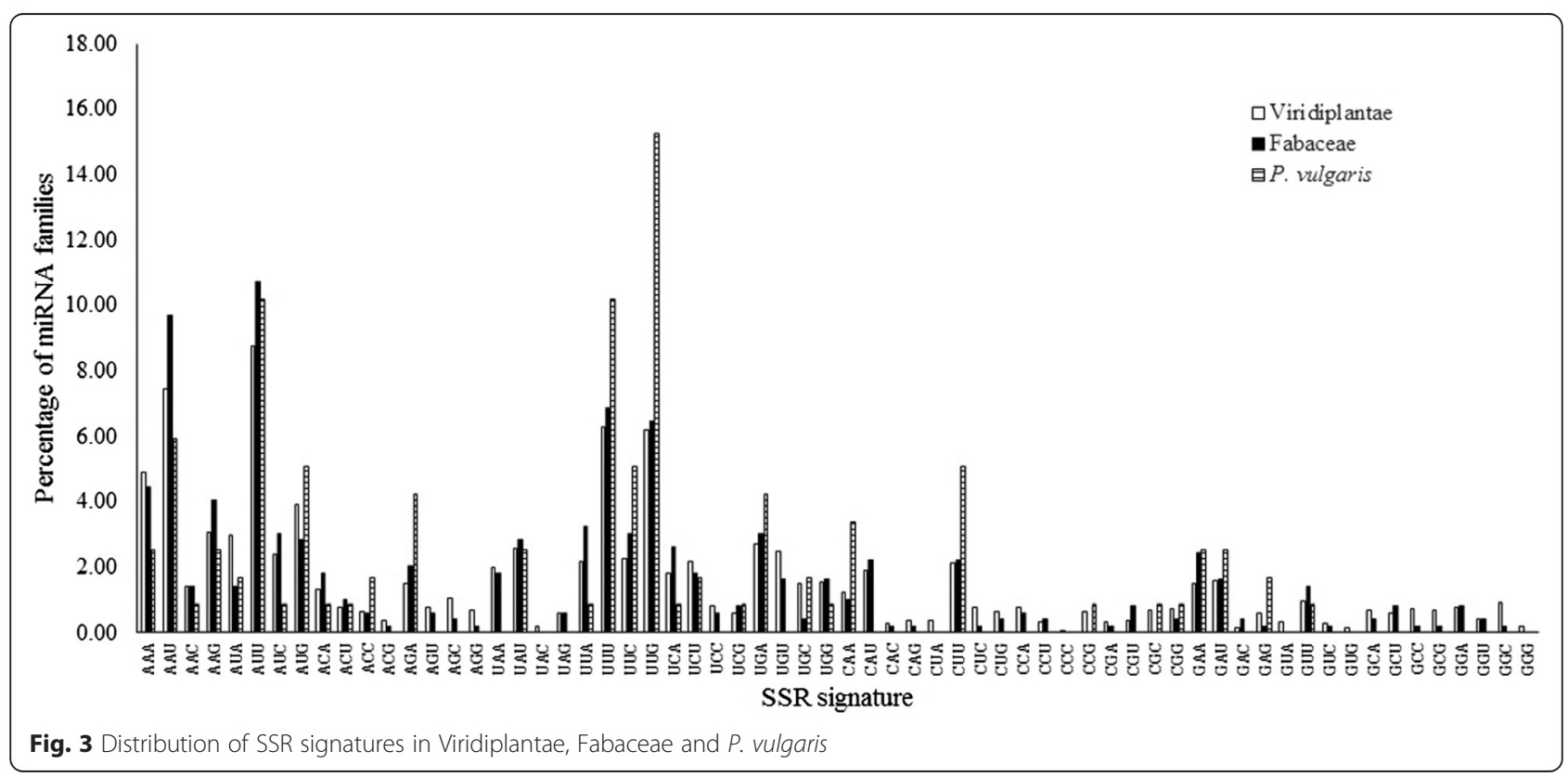


sequences were extracted with all possible lengths. These sequences were used in BLASTX to identify and remove the protein coding sequences. After removal, the number of sequences reduced to $122,163,665$. These sequences were examined for the seven criteria mentioned in the 'materials and methods' section, and only those fulfilling these criteria were retained as the predicted pre-miRs. In case of multiple sequences resulted from a single BLAST hit, the one which fulfils all the seven criteria with the maximum MFEI and the maximum $\mathrm{R}$ was retained. Finally, 310 sequences were obtained and were designated as putative pre-miRs in P. vulgaris. Extraction of the mature miRNAs from these 310 pre-miRs resulted in 208 new miRNAs, of which 201 are novel. These new miRNAs belong to 118 miRNA families in P. vulgaris (Additional file 2 Table S2). Fig. 4 shows a particular miRNA 'pvumiR399a' that fulfils all the seven criteria used for the prediction.

The distribution of 208 newly predicted miRNAs in $P$ vulgaris varies among the 118 miRNA families (Table 2). Four of the families namely MIR1533, MIR1527, MIR5021 and MIR848 are the most populated families with 15, 10, 10 and 7 members, respectively, while 85 families contain only one member. In the remaining 29 families, the number of miRNA varies from 2 to 5 . This is in accordance with the diversity observed in other plant species [42]. The length distribution of newly predicted miRNAs (Fig. 5) shows that the length of mature miRNAs fall within the range of 15-24 nucleotides with an average length of 19 nucleotide $( \pm 1.6)$. However, miRNA pvu-miR848f is the only exception with the length of 14 nucleotides.

\section{Experimental validation of the predicted miRNAs in $P$. vulgaris}

Deep-sequencing of $P$. vulgaris small RNA library generated a total of $33,672,751$ reads. The low quality reads as well the reads with lower than 14 nucleotide length were removed, resulting in 33,602,649 reads. The reads were made unique using fastx_collapser. The sequencing data obtained was BLAST searched with predicted miRNAs. The presence of 97 (Additional file 3 Table S3) of the predicted miRNAs in $P$. vulgaris is confirmed from the sequencing data.

qRT-PCR was used to experimentally validate our computational method and to compare the results with the sequencing data. A total of 5 computationally predicted miRNAs were randomly chosen (Table 3 ) and qRT-PCR was done for these five miRNAs. $\mathrm{C}_{\mathrm{T}}$ values were calculated using U6 snRNA as a normaliser gene. The relative quantity of each miRNA to U6 snRNA was expressed using the formula $2^{-\Delta C T}$ [43], where $\Delta C_{T}=\left(C_{T}\right.$ miRNA $\mathrm{C}_{\mathrm{T}} \mathrm{U} 6$ snRNA) (Fig. 6). The expression profiles obtained by qRT-PCR analysis mostly agreed with the expression values obtained from the sequencing data of these $5 \mathrm{miR}$ NAs (Fig. 7). For pvu-miR1519a, in qRT-PCR, the $\mathrm{C}_{\mathrm{T}}$ value obtained is quite high (34.4) indicating that it is a very low expressed miRNA and this result correlated with the sequencing data where the number of reads of this miRNA is only 2 (TPM 0.06). For pvu-miR5368b, the number of reads obtained from sequencing data is 1290 (TPM 38.4), the same value for pvu-miR5368a also, however, the relative expression obtained in qRT-PCR for pvumiR5368b is lower than that of pvu-miR5368a. This may be due to the fact that pvu-miR5368b expression is relatively low in leaves compare to other tissues. Several studies already have established that miRNA expression can vary widely in different tissues or at different developmental stages $[44,45]$.

\section{Computational validation of the prediction method}

The computational method developed in this study was used to predict the miRNAs of $A$. thaliana and the results were compared with known miRNAs of $A$. thaliana (miRBase 20). The miRNAs from Viridiplantae excluding those from $A$. thaliana and the genome of $A$. thaliana were used as the inputs for prediction pipeline. A total of 229 miRNAs (Additional file 4. Table S4) were predicted, of which 213 are already reported in miRBase 20. The same procedure was repeated for G. max. A total of 462 miRNAs (Additional file 5 Table S5) were predicted, of which 397 are already reported in miRBase 20. The performance of the prediction method is measured using parameters sensitivity, specificity, positive predictive value (PPV) and negative predictive value (NPV). Our computational prediction method has a high sensitivity of 0.97 as well as high specificity of 0.99 (Table 4).

\section{Prediction of the miRNA targets in $P$. vulgaris}

The psRNATarget server was used to predict the miRNA targets. The default sequences of the target candidates in the server are of old version, hence the updated EST sequences of $P$. vulgaris from NCBI GenBank were used as target candidates. For 130 miRNAs that belong to 69 families, 1303 target sequences were predicted. In order

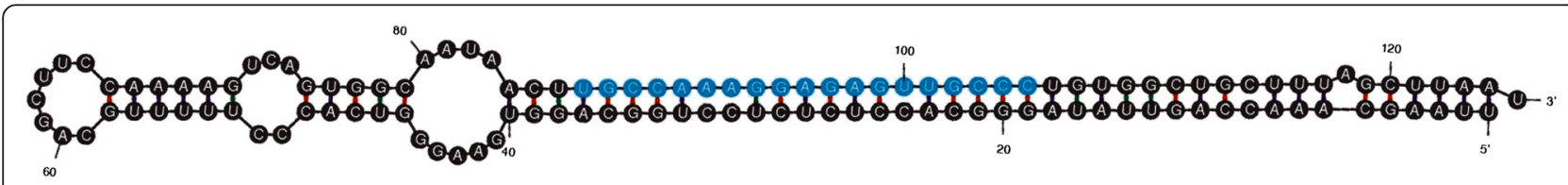

Fig. 4 Secondary structure of a pre-miR (pvu-miR399a) showing the mature miRNA sequence highlighted in blue 
Table 2 Distribution of miRNAs within different miRNA families of $P$. vulgaris

\begin{tabular}{l}
\hline miRNA families \\
\hline MIR1533 \\
MIR1527 \\
MIR5021 \\
MIR848 \\
MIR167, MIR171 \\
MIR156, MIR159, MIR166, MIR169, MIR6034 \\
MIR319, MIR3440, MIR5054, MIR529, MIR5721, MIR6470, MIR902 \\
MIR1514, MIR2606, MIR2673, MIR3442, MIR396, MIR4345, MIR477, MIR5261, MIR5368, MIR5558, MIR5654, MIR5998, \\
MIR6169, MIR829, MIR866 \\
MIR1029, MIR1030, MIR1043, MIR1044, MIR1051, MIR1052, MIR1075, MIR1099, MIR1134, MIR1217, MIR1428, MIR1441, \\
MIR1519, MIR165, MIR1846, MIR1860, MIR1888, MIR1916, MIR2082, MIR2088, MIR2095, MIR2105, MIR2109, MIR2610, \\
MIR2873, MIR2934, MIR2938, MIR3444, MIR3630, MIR3633, MIR3711, MIR395, MIR3954, MIR3979, MIR398, MIR399, \\
MIR408, MIR419, MIR4224, MIR4225, MIR4243, MIR4245, MIR4246, MIR4413, MIR482, MIR5014, MIR5041, MIR5057, \\
MIR5083, MIR5140, MIR5169, MIR5176, MIR5177, MIR5179, MIR5213, MIR5248, MIR5255, MIR5264, MIR5281, MIR5298, \\
MIR5555, MIR5562, MIR5662, MIR5674, MIR5675, MIR5741, MIR5773, MIR5778, MIR5820, MIR6027, MIR6114, MIR6167, \\
MIR6171, MIR6196, MIR6214, MIR6479, MIR6484, MIR771, MIR773, MIR774, MIR831, MIR846, MIR861, MIR863, MIR919
\end{tabular}

to characterise the targets, BLASTX was used with the predicted target sequences as query and the entire protein sequences of Viridiplantae as subject. Using $80 \%$ sequence identity cut-off, 318 targets for 95 miRNAs were characterised (Additional file 6 Table S6). For additional 339 targets for 80 miRNAs, the BLASTX predicted uncharacterised and hypothetical proteins. The hybridized structures of mature pvu-miR166d with its two targets, EST 312062389 coding for UDP-
$\mathrm{N}$-acetyl glucosamine pyrophosphorylase protein and EST 312035414 coding for SNF1-related protein kinase regulatory subunit are shown in Fig. 8.

\section{Discussion}

In the last decade, numerous studies confirmed that plant miRNAs are directly involved in developmental processes such as seed germination, morphogenesis, floral organ identity, root development, vegetative and reproductive

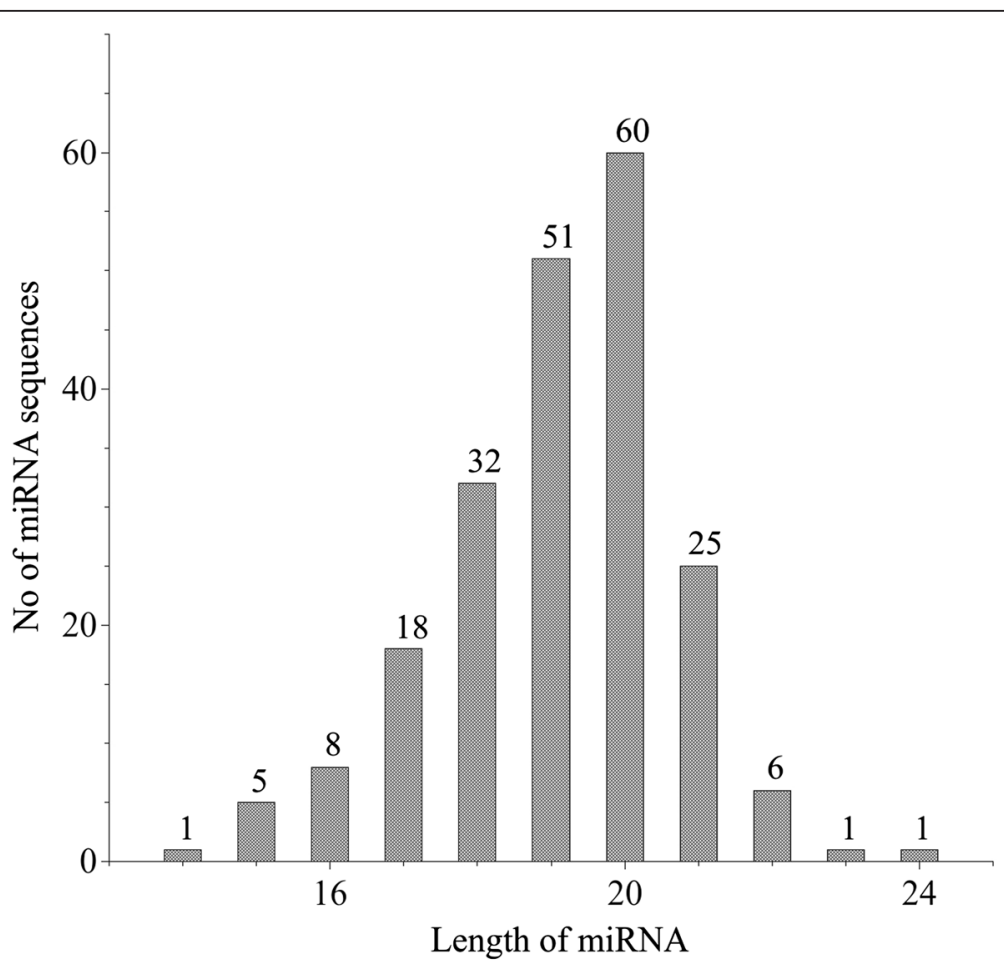

Fig. 5 Frequency distribution of the length of mature miRNAs of $P$. vulgaris 
Table 3 Stem-loop reverse transcription primers for selected miRNAs

\begin{tabular}{lll}
\hline miRNA & miRNA Sequence & Primer sequences \\
\hline pvu-miR1519a & AGUGUUGCAAGAUAGUCAUU & Reverse transcription primer: GTCGTATCCAGTGCAGGGTCCGAGGTATTCGCACTGGATACGACAATGAC \\
& & Forward primer: CGGCGCAGTGTGCAAGA \\
& Universal reverse primer: CCAGTGCAGGGTCCGAGGTA \\
pvu-miR5054b & UGGCGCCCACCGUGGGG & Reverse transcription primer: GTCGTATCCAGTGCAGGGTCCGAGGTATCGCACTGGATACGACCCCCAC \\
& & Forward primer: GGGGCCTGGCGCCCACCG \\
& Universal reverse primer: CCAGTGCAGGGTCCGAGGTA \\
pvu-miR5368a & GGACAGUCUCAGGUAGACA & Reverse transcription primer: GTCGTATCCAGTGCAGGGTCCGAGGTATTCGCACTGGATACGACTGTCTA \\
& & Forward primer: CGGCGCCGGACAGTCTCAGG \\
& & Universal reverse primer: CCAGTGCAGGGTCCGAGGTA \\
pvu-miR5368b & UGUCUACCUGAGACUGUCC & Reverse transcription primer: GTCGTATCCAGTGCAGGGTCCGAGGTATTCGCACTGGATACGACGGACAG \\
& & Forward primer: CGGCGCCTGTCTACCTGAGA \\
& & Universal reverse primer: CCAGTGCAGGGTCCGAGGTA \\
pvu-miR1527j & UAACUCAACCUUAUAAAAC & Reverse transcription primer: GTCGTATCCAGTGCAGGGTCCGAGGTATCGCACTGGATACGACGTTTA \\
& & Forward primer: CGGCGCCTAACTCAACCTTA \\
& Universal reverse primer: CCAGTGCAGGGTCCGAGGTA \\
\hline
\end{tabular}

phase change, flowering initiation and seed production [46-51]. In addition to their important functions in organ development, plant miRNAs play a crucial role at the core of gene regulatory networks. They are involved in various biotic and abiotic stress responses, [52-54] signal transduction and protein degradation [55]. Plant miRNAs also play an important role in the biogenesis of small RNAs (siRNAs) and in the feedback regulation of siRNA pathways.

In the present study, using computational methods, we have identified 208 new miRNAs in P. vulgaris of which
201 are novel. Of these 208 predicted miRNAs, 97 were validated through small RNA sequencing. In general, computational prediction of miRNAs uses a highly constrained search space by setting fixed values to parameters like AU content, MFEI and the length of the pre-miRs $[12,13,15,16]$. Constraining the parameters to a fixed cut-off value reduces the number of predicted miRNAs. It is already an established fact that the commonly used parameters namely the length of pre-miRs, AU content and MFEI are highly variable, ranging between 43-938,

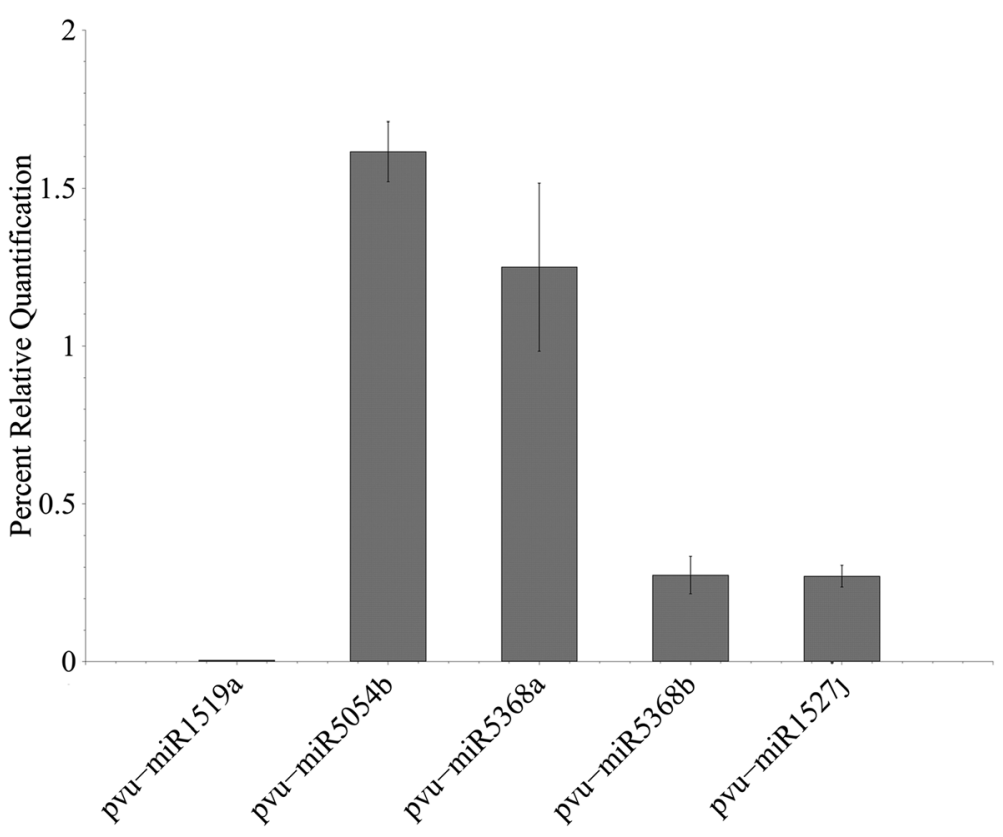

Fig. 6 Expression profile of selected miRNAs from qRT-PCR analysis 


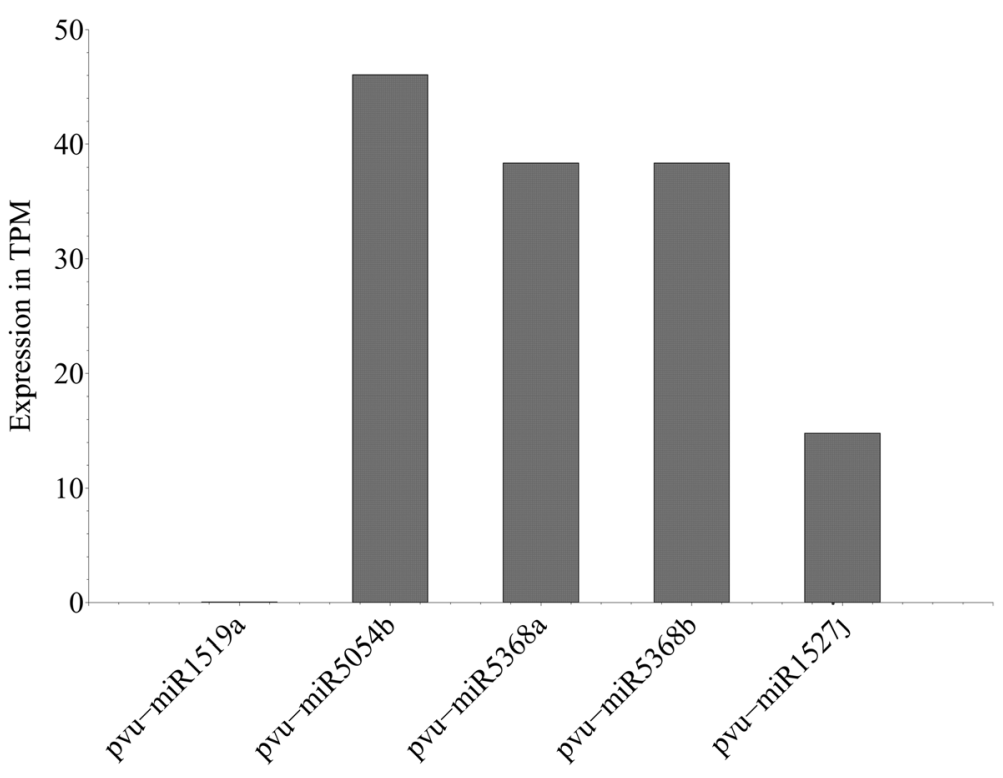

Fig. 7 Expression profile in TPM of selected miRNAs from sequencing data

$17 \%-92 \%$ and $0.32-2.7$, respectively. The distribution of ND, Npb and NQ (Fig. 2) in miRNAs is significantly different from other small RNAs, making them good candidates as prediction parameters. However, there is also an overlapping region in the distribution, which can result in false positives while predicting using single parameter. Thus using a combination of these parameters will make the prediction pipeline more robust. In the present study, instead of using the conventional computational procedure, where all the prediction parameters are set to a fixed value, we have used a $99 \%$ probability range. Initial application of fixed cut-off values for various parameters resulted in only 26 new miRNAs in $P$. vulgaris. This low number of miRNAs prompted us to use the $99 \%$ probability range with the anticipation of getting better prediction. After using the $99 \%$ probability range for the first six parameters described in the 'materials and methods' section, 2538 pre-miRs in $P$. vulgaris were predicted, which is almost hundred times compared to the conventional method. However, it should be noted that the increased number includes both new predictions as well as false positives. False positives are eliminated by using the RNA folding parameters and conserved SSR signature.

Table 4 Statistical parameters to measure accuracy of prediction method

\begin{tabular}{lcc}
\hline Parameter & A. thaliana & G. max \\
\hline Sensitivity & 0.97 & 0.97 \\
Specificity & 0.99 & 0.98 \\
Positive predictive value & 0.93 & 0.86 \\
Negative predictive value & 0.99 & 0.99 \\
\hline
\end{tabular}

The presence of SSRs in pre-miRNAs is already established [29-31], although their specific role in pre-miRs is still unknown. Most of the SSRs in pre-miRs have few steady characteristics, allowing their identification in pre-miRs feasible. Thus conserved SSR signatures are a potential parameter in predicting new miRNAs. In the present study, we have used the conserved SSR signatures as a prediction parameter. By using this parameter, the predicted number of $2538 \mathrm{P}$. vulgaris pre-miRs was reduced to 310 . We have identified the SSR signatures for all the Viridiplantae miRNAs present in the miRBase 20 (Additional file 1 Table S1), and these signatures can be used for the identification of new miRNAs in any species of Viridiplantae.

Along with the SSR, we have also used NQ, ND and $\mathrm{Npb}$ in our prediction. After filtering the putative premiRs through these four parameters, the length, AU content and MFEI for the predicted pre-miRs of $P$. vulgaris vary from $55-105,33-77 \%$ and $0.42-1.2$, respectively. These values are in agreement with known pre-miRs in Viridiplantae. These four independent parameters do not restrict the physical and thermodynamic features of premiRs to fixed values, and can be used for successful prediction of new miRNAs in plants.

The miRBase 20 contains 7385 mature miRNAs of Viridiplantae. Analysis of these 7385 miRNAs revealed that more than $70 \%$ of them belong to the 13 wellstudied plant species namely Medicago truncatula, Oryza sativa, Glycine max, Brachypodium distachyon, Populus trichocarpa, Arabidopsis lyrata, Solanum tuberosum, Arabidopsis thaliana, Zea mays, Physcomitrella patens, Sorghum bicolor, Prunus persica and Malus domestica. Further we find that, each of these 13 species have more 


\section{(a)}

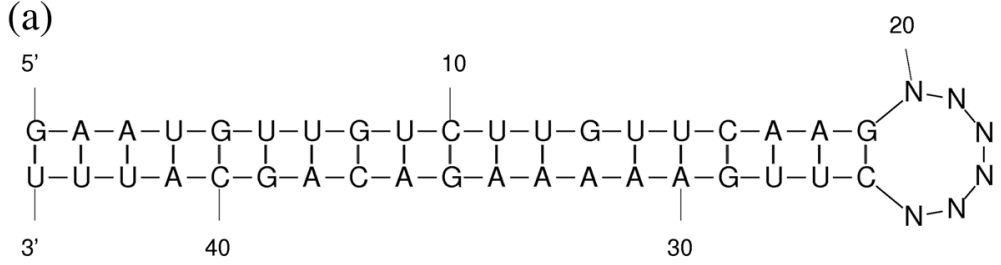

(b)

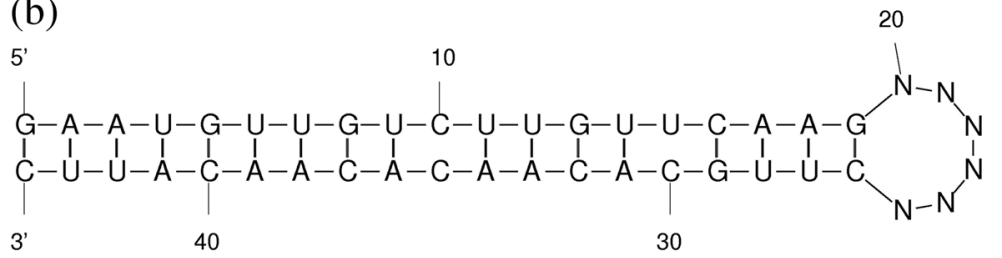

Fig. 8 Hybridized structure of mature miRNA with its targets. The mature miRNA forms the $5^{\prime}$ end and the target is at the $3^{\prime}$ end separated by 6 nucleotides. The pvu-miR166d with its two targets: (a) EST 312062389 coding for UDP-N-acetylglucosamine pyrophosphorylase protein regulated by cleavage, (b) EST 312035414 coding for SNF1-related protein kinase regulatory subunit inhibited by translational regulation

than 200 mature miRNAs reported in the miRBase. In the present study, prediction of the 208 mature miRNAs in $P$. vulgaris is in accordance with this finding, thus justifying our modified computational prediction method.

In order to validate the computationally predicted miRNAs, small RNA library was prepared from the Anupam cultivar of $P$. vulgaris. The quality reads with more than 14 nucleotide length were BLAST searched with the predicted miRNAs. Out of the 208 predicted miRNAs, 97 are expressed in the sequenced sample. The read numbers for miRNAs showed high diversity, ranging from 1 to 37,259 for the expressed miRNAs. Among these miRNAs, the miR166 family had the most number of reads. For all the identified miRNAs, transcript per million (TPM) was also calculated. The dataset of known pre-miRs downloaded from the miRBase 20 contains miRNAs deposited from different cultivars of $P$. vulgaris at different developmental stages. However, the small RNA library created for sequencing is from a single cultivar of $P$. vulgaris at a particular stage of development, which makes it impossible for all the predicted 208 miRNAs to be present in the sequence library. The presence of nearly fifty percent of the predicted miRNAs in the sequencing data justifies our method followed in computational prediction of miRNAs.

Additionally, five randomly selected computationally predicted miRNAs were validated using qRT-PCR. Relative expressions obtained in the qRT-PCR mostly corroborated the sequencing data; only slight variation for pvu-miR5368b can be attributed to the fact that miRNA expression widely varies in different tissues and this particular miRNA may have relatively low expression in leaf tissues. The validation of the five randomly selected predicted miRNAs in both qRT-PCR and Illumina sequencing substantiate our computational method for the prediction of miRNAs.

All the newly predicted 208 miRNAs in P. vulgaris belong to 118 miRNA families. We find that of these 118 families, only 15 contain miRNAs distributed into 10 plant species. Although, these miRNA families have a wide species range, yet low number of miRNAs are present from the species of Fabaceae family (Table 5). There are 21 miRNA families containing a single miRNA from one of the species of Fabaceae, showing the under representation of miRNAs of Fabaceae in the miRBase. Fabaceae, one of the most important families in the Dicotyledonae [56], is rich in high quality protein, providing high nutritional food crops for agriculture all over the world. Our prediction of 208 new miRNAs in P. vulgaris as well as identification and characterisation of their targets will enrich the present knowledge of Fabaceae miRNAs, and will definitely help in deciphering the role of miRNAs in different regulatory mechanisms.

miRBase 20 contains 427 mature miRNAs of $A$. thaliana of which 220 homologs are present in other species of Viridiplantae. The rest of the known miRNAs (207) from $A$. thaliana have no known homolog in other plant species, making them difficult to predict. We have also predicted 213 miRNAs of the known homologs from a total prediction of 229 miRNAs in A. thaliana. Besides, we also predicted 462 miRNAs in G. max of which 397 exists in miRBase 20 (97\% of 408 reported miRNAs). This successful prediction not only validates our method, but also establishes that the method can be applied to predict the miRNAs in any other plant species.

The prediction method can be evaluated using various statistical parameters such as sensitivity, specificity, PPV and NPV. Sensitivity measures the proportion of miRNAs which are correctly identified by the prediction pipeline, 
Table 5 Distribution of Fabaceae species in various miRNA

\begin{tabular}{|c|c|c|}
\hline $\begin{array}{l}\text { miRNA } \\
\text { Family } \\
\end{array}$ & Number of Viridiplantae species & Number of Fabaceae species \\
\hline 156 & 48 & 3 \\
\hline 159 & 35 & 2 \\
\hline 166 & 42 & 3 \\
\hline 167 & 37 & 4 \\
\hline 169 & 36 & 3 \\
\hline 171 & 41 & 4 \\
\hline 319 & 34 & 6 \\
\hline 395 & 30 & 3 \\
\hline 396 & 42 & 5 \\
\hline 398 & 30 & 2 \\
\hline 399 & 30 & 4 \\
\hline 408 & 32 & 5 \\
\hline 482 & 23 & 5 \\
\hline 529 & 10 & 1 \\
\hline 1514 & 2 & 2 \\
\hline 1519 & 1 & 1 \\
\hline 1527 & 1 & 1 \\
\hline 1533 & 1 & 1 \\
\hline 2088 & 1 & 1 \\
\hline 2109 & 2 & 2 \\
\hline 2606 & 2 & 2 \\
\hline 2610 & 1 & 1 \\
\hline 2673 & 1 & 1 \\
\hline
\end{tabular}

whereas specificity measures the proportion of sequences which are correctly rejected. Our prediction method shows both high sensitivity and specificity when tested for known miRNAs of $A$. thaliana and G. $\max$ (Table 4). The parameters PPV and NPV measures the probability of predicted or rejected sequences to be true miRNAs or not, respectively. Higher values of PPV and sensitivity give us a high confidence for a positive prediction, while higher values of NPV and specificity give us high confidence for the rejection

Recently, numerous studies suggested that the genomic distribution of SSRs are nonrandom, and the SSRs located in gene or regulatory regions play important role in chromatin organization, regulation of gene activity, recombination, DNA replication, cell cycle, mismatch repair system $[57,58]$. The transcriptome survey of several plant species showed the high abundance of di- and trinucleotide repeats compare to tetra-, penta- and hexa nucleotide repeats; $(\mathrm{AT})_{\mathrm{n}}$ repeat being the most frequently occurring microsatellites in plant genomes [59-63]. The microsatellites in the genomic sequences play vital role in the biogenesis of several small non-coding RNAs, of which most important are the miRNAs. Transcriptome analysis of several plants revealed that a significant percentage of the unigenes constitutes 'SSR bearing premiRNA candidates' [58], suggesting that SSRs are an important component of pre-miRs. SSRs in pre-miRs are derived from independent transcriptional units and often relate to function [32]. Variations of SSRs within pre-miRs are very critical for normal miRNA activity as expansion or contraction of SSRs in pre-miRs directly affects the corresponding miRNA products and may cause unpredicted changes [32]. These characteristics features foster exploit of SSR signature as a critical parameter in miRNA identification [32]. The number of miRNAs predicted in the traditional method is too low and we have introduced $99 \%$ probability region for increasing the search space. However, this has increased the number of false predictions. As a result of this, the number of miRNAs predicted before the SSR filtering step for A. thaliana and G. $\max$ are 2082 and 3541, respectively. In spite of these high numbers of predictions, by using SSR the final numbers of predicted miRNAs were restricted to 229 and 462, respectively in these two species. The specificity of our prediction method improved from 0.62 to 0.99 in A. thaliana and 0.49 to 0.98 in G. max, by applying SSR filtration step. Thus SSR signatures act as an effective filtering parameter in limiting the number of false positives to acceptable limits.

The mature miRNA sequences and EST sequences of $P$. vulgaris were submitted to the psRNATarget server for the prediction of targets. The parameters were adjusted as described in 'materials and methods' section for better prediction. The hpsize [64] was changed according to the length of miRNA, as the server uses a value assuming the length of miRNA as 20 nucleotides. The miRNAs with length lesser than hpsize were ignored by the server pipeline. The length of the miRNAs predicted in the present study varies from 14-24 nucleotides. The sequence length of central mismatch was also changed according to the length of the miRNA. This parameter helps to predict the targets inhibited by translational regulation and has no effect on targets inhibited by cleavage of mRNA sequence [65]. Further, the maximum expectation value was set to 2.0 for stringent filtering of false positive targets predicted by the server.

In the present study, 1305 targets were predicted for 130 miRNAs. Of these 1305 targets, functional information was retrieved for 318 targets distributed in 46 miRNA families. In majority of the cases, the predicted targets in this study were in accordance with the already published reports in other plant species. Yu et al. [66] showed that miR156 family control plant development by regulating the trichome growth in Arabidopsis. It is already established that MYB transcription factors are the 
negative controllers of the trichome growth. The miR156 family targets the MYB transcription factor mRNAs, and by cleaving these transcription factors they positively control the trichome growth. We also found that the predicted pvu-miR156d target the MYB transcription factors. In the present study pvu-miR166d was predicted to target kinase mRNA, which is in agreement with the reported target kinase for miR166 family in soybean [67]. Calvino and Messing [68] established that miR169 family in Sorghum targets the carboxypeptidase mRNAs. Similarly, in the present study, pvu-miR169b was predicted to target the carboxyl-terminal-processing protease. Scarecrow-like transcription factor is already an established target for miR171 family in Arabidopsis [69] and Oryza sativa [70]. Similar results were obtained in our study where pvumiR171a was predicted to bind Scarecrow-like transcription factor. ATP sulfyrylase responsible for sulphur (S) uptake and assimilation is the target for miR395 family in Arabidopsis [69], rice [70] and soybean [67]. Newly identified pvu-miR395a was also predicted to target the ATP sulfyrylase. In Arabidopsis, it was found that miR396 family targets the tubulin mRNAs [71]. Our prediction was in accordance with this finding, showing that pvu-miR396b targets gamma tubulin. Basic blue proteins (Plantacyanins) are validated targets for miR408 family in Arabidopsis and rice [70, 72]. Similar target was predicted for pvu-miR408a. The predicted target fatty acid desaturase of pvu-miR902c in our study is in agreement with the findings of Wan et al. [73] showing that the targets of miR902 are primarily involved in lipid metabolism.

\section{Conclusion}

In this study, we have used computational method to identify new miRNAs in $P$. vulgaris and few of them were experimentally validated. We have used conserved SSR signatures to predict new miRNAs. We have identified 208 new miRNAs belonging to 118 different families of miRNAs in P. vulgaris, of which 201 are novel. We have also predicted 1305 targets for 130 of these miRNAs. We successfully predicted known miRNAs in A. thaliana and G. max using our method. Presently, numerous miRNAs from various plant species have been identified and characterized by the aid of next-generation sequencing. However, there is still inadequate information of miRNAs in many plant species. Identification of new miRNAs in all plant species and deciphering their functions is the present day challenge in biological discoveries. Wet-lab experiments have their own limitations and the alternate approach is in silico methods for miRNA studies. In silico methods can rapidly identify new miRNAs and their targets in any species. The computational approach that we have developed can be successfully applied to identify new miRNAs and their targets in any plant species, and is expected to generate an optimal framework for deciphering the biogenesis, functions, and mechanisms of plant miRNAs that are not yet discovered.

\section{Methods}

\section{Data collection and preparation}

The Viridiplantae pre-miRs were downloaded from the miRBase 20 (Release 20: June 2013) [74] and used as the standard dataset of known pre-miRs. The small RNAs belonging to different families were downloaded from Rfam 11 [75] for comparative analysis of various parameters. The miRBase 20 contains 24,521 pre-miRs, of which 6088 belong to Viridiplantae. Besides, we have also downloaded 125,490 Expressed Sequence Tags (ESTs) and 92,534 Genomic Survey Sequences (GSSs) of P. vulgaris (txid3885) from the GenBank [76]. Removal of the redundant sequences resulted in 2560 Viridiplantae pre-miRs, and 122,157 EST and GSS sequences of P. vulgaris. Protein sequences of $P$. vulgaris were downloaded from the protein database (http://www.ncbi.nlm.nih.gov/protein). Genomes of $A$. thaliana [77] and G. $\max$ [78] were downloaded from Phytozome [79].

\section{Analysis of known precursor sequences}

All the downloaded 6088 Viridiplantae pre-miRs were used to calculate the length of pre-miRs sequences (L), AU content and MFEI. The structures with the minimum folding energy was generated using RNAfold [80]. The MFEI value was calculated using the Adjusted MFE (AMFE), which represents the MFE for 100 nucleotides.

$$
\begin{aligned}
& A M F E=\frac{-M F E}{\mathrm{~L}} \times 100 \\
& M F E I=\frac{A M F E}{(G+C) \%}
\end{aligned}
$$

The genRNAstats program [81] was used to calculate the NQ, ND and Npb for all known pre-miRs of Viridiplantae. Npb is the measure of total number of base pairs present in the RNA secondary structure per length of the sequence, and the value can range from 0.0 (no base-pairs) to 0.5 (L/2 base-pairs) [82]. The base-pairing probability distribution (BPPD) per base in a sequence were measured using NQ [83], while the base-pair distance for all the pair of structures were measured using ND [84]. Both the parameters ND and NQ were calculated from the base-pair probability $\mathrm{p}_{\mathrm{ij}}$ between bases $\mathrm{i}$ and $\mathrm{j}$.

$$
N Q=-\frac{1}{L} \sum_{i<j} p_{i j} \cdot \log _{2}\left(p_{i j}\right)
$$




$$
N D=\frac{1}{L} \sum_{i<j} p_{i j}\left(1-p_{i j}\right)
$$

The miRBase 20 classified Viridiplantae miRNAs into 1892 families. We have checked the presence of conserved SSR signatures within the pre-miRs of all these families. The conserved SSR signatures were counted in all sequences for window size ranging from three to six. Due to the variable length of the pre-miRs, the SSR signatures were normalized per 100 nucleotides by the following equation.

$$
R=\frac{\text { Number of SSR signatures }}{\mathrm{L}} \times 100
$$

\section{Prediction of new miRNAs in $P$. vulgaris}

BLAST search [85] was performed using the nonredundant dataset of Viridiplantae pre-miRs as query and non-redundant dataset of EST and GSS sequences of $P$. vulgaris as subject, with an e-value cut-off of 1000 , word size 7 and mismatch less than 4 [86]. The upstream and (or) downstream sequences with all possible lengths ranging from 55-505 were extracted from EST and GSS sequences that aligned with the miRNAs. In order to remove the protein coding sequences, an ungapped BLASTX with the sequence identity cut-off $\geq 80 \%$ was performed with all the extracted sequences as query and the protein sequences of $P$. vulgaris as subject. After removal of the protein coding sequences, remaining sequences satisfying the following criteria were designated as the predicted precursor sequences: (i) formation of an appropriate stem-loop hairpin secondary structure with minimum free energy of folding and MFEI $\geq 0.41$, (ii) a mature miRNA sequence located in one arm of the hairpin structure, (iii) miRNA sequence having less than 6 mismatches with the opposite miRNA* sequence on the other arm of the hairpin structure, (iv) without any loop or break in miRNA* sequence, (v) AU content of the sequences within the range $22-77 \%$, (vi) values for the parameters $\mathrm{NQ}, \mathrm{ND}$ and $\mathrm{Npb}$ should be $\leq 0.45, \leq 0.15$ and $\geq 0.25$, respectively and (vii) presence of SSR signature in the corresponding miRNA family with $R \geq 2.5$. In case of multiple sequences resulted from a single BLAST hit, the particular sequence that fulfils all the above seven criteria along with the maximum values of MFEI and $R$ was chosen. The above mentioned steps are presented in a schematic diagram in Fig. 9. Mature miRNAs were extracted from the predicted pre-miRs satisfying the above criteria.

\section{Experimental validation of predicted miRNAs of $P$. vulgaris Plant material}

Healthy seeds of Anupam cultivar of $P$. vulgaris were surface sterilized with $0.5 \%$ Sodium hypochlorite and germinated in dark at $28{ }^{\circ} \mathrm{C}$ for 2 days. Germinated seeds were allowed to grow in soilrite in BOD at $24{ }^{\circ} \mathrm{C}$ for 10 days. Seedlings of 10 days old were sent in RNAlater (Sigma-Aldrich) to Genotypic-Bangalore, India, for further library preparation and sequencing.

\section{Sequencing}

Library preparation was performed at Genotypic Technology's Genomics facility following certified protocols from NEXT Flex. Small RNA libraries for sequencing were constructed according to the NEXTflex ${ }^{\mathrm{min}}$ small RNA library protocol outlined in NEXTflex ${ }^{\text {Tw }}$ Small RNA Sequencing Kit - 5132-02. $1.6 \mu \mathrm{g}$ of total RNA was used as the starting material. Briefly, 3 ' adaptors were ligated to the specific $3{ }^{\prime} \mathrm{OH}$ group of small RNA followed by $5^{\prime}$ adaptor ligation. The ligated products were reverse transcribed by priming with reverse transcriptase primers. The cDNA was enriched by PCR (12 cycles) and size selection was done using $8 \%$ polyacrylamide gel. The library was size selected in the range of $140-160 \mathrm{bp}$, followed by overnight gel elution and salt precipitation using glycogen, $3 \mathrm{M}$ sodium acetate and absolute ethanol. The precipitate was re-suspended in resuspension buffer. The prepared library was quantified using Qubit fluorometer, and validated for quality by running an aliquot on high sensitivity Bioanalyzer Chip (Agilent). The Bioanalyzer profiles showing fragments between $\sim 130$ to $\sim 160 \mathrm{bp}$ with insert size being $\sim 10$ to $\sim 40$ bp were sent for sequencing.

\section{Sequencing data analysis}

The quality reads from the sequencing data were extracted and the adapter sequences were removed using cutadapt [87]. The sequences smaller than 14 nucleotides were removed. The reads were made unique for easy analysis by using fastx_collapser (http://hannonlab.cshl.edu/ fastx_toolkit/index.html). The predicted miRNAs of $P$. vulgaris were BLAST searched against the sequencing data to validate the predictions.

\section{Small RNA isolation}

Small RNA was isolated from leaves of 10 days old seedlings of Anupam cultivar using mirPremier microRNA isolation kit (Sigma-Aldrich) according to the manufacturer's instruction. The quality and quantity of the isolated small RNA was measured using a microvolume spectrophotometer (JENWAY 7310) and stored at $-20{ }^{\circ} \mathrm{C}$.

\section{cDNA synthesis and primer design}

Small RNA was reverse transcribed to cDNA using stemloop reverse transcription primers for miRNAs as listed in Table 3 following a pulsed RT reaction [88]. Stem-loop primers, forward and reverse primers were designed according to Kramer [89]. A three step pulsed RT reaction was performed; an initial denaturation step at $80^{\circ}$ 


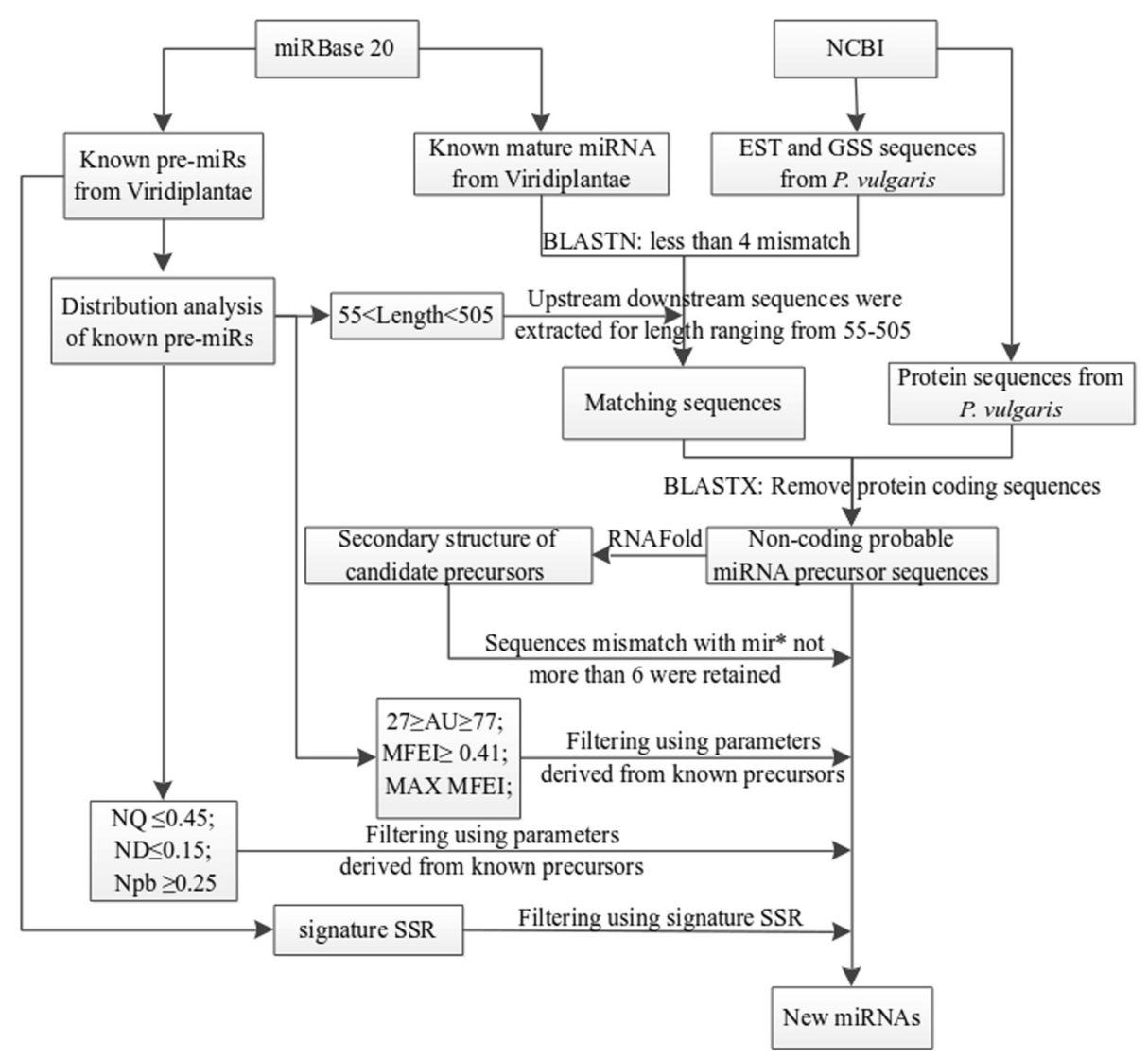

Fig. 9 Schematic representation of the computational method followed in the prediction of new miRNAs in P. vulgaris

C for 5 min containing $20 \mathrm{ng}$ of small RNA and $1 \mu \mathrm{M}$ of each gene specific primers, followed by primer annealing incubation step at $60{ }^{\circ} \mathrm{C}$ for $5 \mathrm{~min}$, followed by final addition of reaction mixture containing $500 \mu \mathrm{M}$ dNTP, 1X buffer, RNase inhibitor and enhanced avian reverse transcriptase (Sigma-Aldrich).

Quantitative Real Time Polymerase Chain Reaction ( $q R T-P C R$ ) qRT-PCR reactions were carried out for the five selected miRNAs in a Bio-Rad CFX96 Real-Time PCR system using Bio-Rad iQ SYBR green supermix. Gradient PCR $\left(50{ }^{\circ} \mathrm{C}-60{ }^{\circ} \mathrm{C}\right.$ ) was performed to select the ideal annealing temperature of $58{ }^{\circ} \mathrm{C}$ for the amplification of U6 snRNA (endogenous control) and selected miRNAs. The reaction mixture containing $1 \mathrm{X}$ SYBR green supermix, $350 \mathrm{nM}$ of each gene specific forward and reverse primers and cDNA (100 ng) was then incubated at $95{ }^{\circ} \mathrm{C}$ for $2 \mathrm{~min}$., followed by 40 cycles of $95{ }^{\circ} \mathrm{C}$ for $10 \mathrm{~s}$ and $58{ }^{\circ} \mathrm{C}$ for $20 \mathrm{~s}$. Melting curve analysis was carried out to verify the specificity of each amplicons. Each amplification reaction was done in triplicate and the specificity of amplicons was confirmed by the presence of a single peak. Standard curve was prepared for U6 snRNA using a twofold dilution.

\section{Computational validation of the prediction method}

The computational method developed in this study to predict miRNAs was validated by predicting known miRNAs in A. thaliana and G. $\max$. The known miRNAs (excluding those from $A$. thaliana) from Viridiplantae and

Table 6 Adjusted parameters for miRNA target prediction using psRNATarget server

\begin{tabular}{lcc}
\hline $\begin{array}{l}\text { Length of } \\
\text { miRNA }\end{array}$ & $\begin{array}{l}\text { Length for complementarity } \\
\text { scoring }\end{array}$ & $\begin{array}{l}\text { Range of central mismatch } \\
\text { leading to translational } \\
\text { inhibition }\end{array}$ \\
\hline 14 & 14 & $6-8$ \\
15 & 15 & $7-8$ \\
16 & 16 & $7-9$ \\
17 & 17 & $8-9$ \\
18 & 18 & $8-10$ \\
19 & 19 & $9-10$ \\
20 & 20 & $9-11$ \\
21 & 21 & $10-11$ \\
22 & 22 & $10-12$ \\
23 & 23 & $11-12$ \\
24 & 24 & $11-13$ \\
\hline
\end{tabular}


the genome of $A$. thaliana were used as inputs for validation. Similar procedure was followed for G. $\max$. The sensitivity, specificity, PPV and NPV for the prediction was calculated from true positives (TP), true negatives (TN), false positives (FP) and false negatives (FN) using following equations.

$$
\begin{aligned}
& \text { Sensitivity }=\frac{T P}{T P+F N} \\
& \text { Specificity }=\frac{T N}{T N+F P} \\
& P P V=\frac{T P}{T P+F P} \\
& N P V=\frac{T N}{T N+F N}
\end{aligned}
$$

\section{Target prediction of new miRNAs}

The targets for mature miRNAs were predicted using psRNATarget server [90] by submitting the mature miRNAs as query and the EST sequences of $P$. vulgaris as subject. To reduce the number of false predictions, the maximum expectation threshold was set to a stringent value of 2.0. The nucleotides for complementarity scoring, hpsize [64] were selected as equal to the length of the mature miRNAs. The maximum energy of unpairing (UPE) the target site was set as $25 \mathrm{kcal}$ [64]. The flanking length around the target site was selected as 17 nucleotides upstream and 13 nucleotides downstream [91]. Due to the variable length of the mature miRNAs, the sequence range of the central mismatch was adjusted (Table 6). To predict the function of the target sequences, BLASTX was performed against the protein database of the Viridiplantae using $80 \%$ sequence identity cut-off.

\section{Availability of supporting data}

The data set supporting the results of this article is available in the NCBI's Gene Expression Omnibus (GEO) database [92] under accession number GSE68305 (http:// www.ncbi.nlm.nih.gov/geo/query/acc.cgi?acc=GSE68305).

\section{Additional files}

Below is the link to the electronic supplementary material.

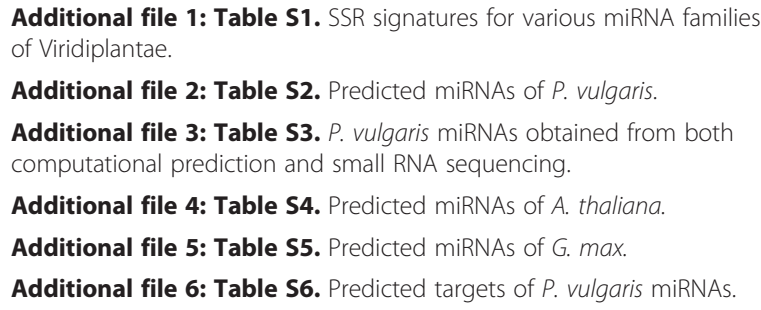

Additional file 1: Table S1. SSR signatures for various miRNA families of Viridiplantae.

Additional file 2: Table S2. Predicted miRNAs of $P$. vulgaris

Additional file 3: Table S3. $P$. vulgaris miRNAs obtained from both computational prediction and small RNA sequencing.

Additional file 4: Table S4. Predicted miRNAs of A. thaliana.

Additional file 5: Table S5. Predicted miRNAs of G. max.

Additional file 6: Table S6. Predicted targets of $P$. vulgaris miRNAs.

\section{Abbreviations}

miRNAs: microRNAs; SSR: Simple sequence repeat; qRT-PCR: Quantitative Real-time polymerase chain reaction; MFEl: Minimal Folding free Energy
Index; pre-miRs: miRNA precursor sequences; RISC: RNA-induced silencing complex; NGS: Next-Generation Sequencing; EST: Expressed Sequence Tags; NQ: Normalised Shannon entropy; ND: Normalized base-pair distance; Npb: Normalized base-pairing propensity; PPV: Positive predictive value; NPV: Negative predictive value; TP: True positives; TN: True negatives; FP: False positives; FN: False negatives.

\section{Competing interests}

The authors declare that they have no competing interests.

\section{Authors' contributions}

RPB and JB conceived the study and participated in its design and coordination. NC and AT performed the analysis of known miRNAs, developed the new algorithm for prediction and predicted the new miRNAs and their targets. NP performed the experimental validation. All the authors participated in the preparation of the final manuscript. All authors read and approved the final manuscript.

\section{Acknowledgements}

We thank the Department of Biotechnology for the research grant (No:BT/PR2680/AGR/36/703/2011) to JB and RPB

Received: 28 January 2015 Accepted: 29 April 2015

Published online: 12 June 2015

\section{References}

1. Ambros V. microRNAs: tiny regulators with great potential. Cell. 2001;107(7):823-6.

2. Bartel DP. MicroRNAs: genomics, biogenesis, mechanism, and function. Cell. 2004;116(2):281-97.

3. Mallory AC, Vaucheret $\mathrm{H}$. Functions of microRNAs and related small RNAs in plants. Nat Genet. 2006:38 Suppl:S31-36.

4. Bushati N, Cohen SM. microRNA Functions. Annu Rev Cell Dev Biol. 2007;23(1):175-205.

5. Carrington JC, Ambros V. Role of microRNAs in plant and animal development. Science (New York, NY). 2003;301(5631):336-8.

6. Djuranovic S, Nahvi A, Green R. A Parsimonious Model for Gene Regulation by miRNAs. Science (New York, NY). 2011;331(6017):550-3.

7. Brennecke J, Stark A, Russell RB, Cohen SM. Principles of MicroRNA-Target Recognition. PLoS Biol. 2005;3(3):e85.

8. Kidner CA, Martienssen RA. The developmental role of microRNA in plants. Curr Opin Plant Biol. 2005;8(1):38-44.

9. Lee RC, Feinbaum RL, Ambros V. The C. elegans heterochronic gene lin-4 encodes small RNAs with antisense complementarity to lin-14. Cell. 1993;75(5):843-54

10. Grad Y, Aach J, Hayes GD, Reinhart BJ, Church GM, Ruvkun G, et al. Computational and Experimental Identification of $C$ elegans microRNAs Molecular. Cell. 2003;11(5):1253-63.

11. Ye K, Chen $Y$, Hu X, Guo J. Computational identification of microRNAs and their targets in apple. Genes Genom. 2013;35(3):377-85.

12. Patanun $O$, Lertpanyasampatha M, Sojikul P, Viboonjun U, Narangajavana J. Computational Identification of MicroRNAs and Their Targets in Cassava (Manihot esculenta Crantz.). Mol Biotechnol. 2013;53(3):257-69.

13. Qiu CX, Xie FL, Zhu YY, Guo K, Huang SQ, Nie L, et al. Computational identification of microRNAs and their targets in Gossypium hirsutum expressed sequence tags. Gene. 2007;395(1-2):49-61.

14. Han Y, Luan F, Zhu H, Shao Y, Chen A, Lu C, et al. Computational identification of microRNAs and their targets in wheat (Triticum aestivum L.). Sci China Ser C-Life Sci. 2009;52(11):1091-100.

15. Xie FL, Huang SQ, Guo K, Xiang AL, Zhu YY, Nie L, et al. Computational identification of novel microRNAs and targets in Brassica napus. FEBS Lett. 2007;581(7):1464-74.

16. Lu Y, Yang X: Computational Identification of Novel MicroRNAs and Their Targets in Vigna unguiculata. Comp Funct Genom. 2010;2010. http://dx.doi.org/10.1155/2010/128297.

17. Jones-Rhoades MW, Bartel DP. Computational identification of plant microRNAs and their targets, including a stress-induced miRNA. Mol Cell. 2004;14(6):787-99.

18. Wang XJ, Reyes $J$, Chua NH, Gaasterland T. Prediction and identification of Arabidopsis thaliana microRNAs and their mRNA targets. Genome Biol. 2004;5(9):R65. 
19. Wang J, Yang $X, X u H$, Chi $X$, Zhang M, Hou X. Identification and characterization of microRNAs and their target genes in Brassica oleracea. Gene. 2012;505(2):300-8.

20. Galla G, Volpato M, Sharbel T, Barcaccia G. Computational identification of conserved microRNAs and their putative targets in the Hypericum perforatum L flower transcriptome. Plant Reprod. 2013;26(3):209-29.

21. Gao Z, Luo X, Shi T, Cai B, Zhang Z, Cheng Z, et al. Identification and validation of potential conserved microRNAs and their targets in peach (Prunus persica). Mol Cells. 2012;34(3):239-49.

22. Song C, Jia Q, Fang J, Li F, Wang C, Zhang Z. Computational identification of citrus microRNAs and target analysis in citrus expressed sequence tags. Plant Biol. 2010;12(6):927-34.

23. Dong Q-H, Han J, Yu H-P, Wang C, Zhao M-Z, Liu H, et al. Computational Identification of MicroRNAs in Strawberry Expressed Sequence Tags and Validation of Their Precise Sequences by miR-RACE. J Hered. 2012;103(2):268-77.

24. Li Y, Li W, Jin Y-X. Computational Identification of Novel Family Members of MicroRNA Genes in Arabidopsis thaliana and Oryza sativa. Acta Biochim Biophys Sin. 2005;37(2):75-87.

25. Lai E, Tomancak P, Williams R, Rubin G. Computational identification of Drosophila microRNA genes. Genome Biol. 2003;4(7):R42.

26. Jia H, Osak M, Bogu GK, Stanton LW, Johnson R, Lipovich L. Genome-wide computational identification and manual annotation of human long noncoding RNA genes. RNA. 2010;16(8):1478-87.

27. Zhang BH, Pan XP, Cox SB, Cobb GP, Anderson TA. Evidence that miRNAs are different from other RNAs. Cell Mol Life Sci. 2006:63(2):246-54.

28. Chen M, Tan Z, Jiang J, Li M, Chen H, Shen G, et al. Similar distribution of simple sequence repeats in diverse completed Human Immunodeficiency Virus Type 1 genomes. FEBS Lett. 2009;583(17):2959-63.

29. Joy N, Asha S, Mallika V, Soniya EV. De novo transcriptome sequencing reveals a considerable bias in the incidence of simple sequence repeats towards the downstream of 'Pre-miRNAs' of black pepper. PLoS One. 2013;8(3):e56694.

30. Joy N, Soniya EV. Identification of an miRNA candidate reflects the possible significance of transcribed microsatellites in the hairpin precursors of black pepper. Funct Integr Genomics. 2012;12(2):387-95.

31. Mondal TK, Ganie SA: Identification and characterization of salt responsive miRNA-SSR markers in rice (Oryza sativa). Gene 2014:535(2):204-209.

32. Chen M, Tan Z, Zeng G, Peng J. Comprehensive Analysis of Simple Sequence Repeats in Pre-miRNAs. Mol Biol Evol. 2010;27(10):2227-32.

33. Rondon M, Lehmann J, Ramírez J, Hurtado M. Biological nitrogen fixation by common beans (Phaseolus vulgaris L.) increases with bio-char additions. Biol Fertil Soils. 2007;43(6):699-708.

34. Arenas-Huertero C, Pérez B, Rabanal F, Blanco-Melo D, la Rosa C, EstradaNavarrete G, et al. Conserved and novel miRNAs in the legume Phaseolus vulgaris in response to stress. Plant Mol Biol. 2009;70(4):385-401.

35. Subramanian S, Fu Y, Sunkar R, Barbazuk WB, Zhu J-K, Yu O. Novel and nodulation-regulated microRNAs in soybean roots. BMC Genomics. 2008;9(1):160

36. Sunkar $\mathrm{R}$, Jagadeeswaran $\mathrm{G}$. In silico identification of conserved microRNAs in large number of diverse plant species. BMC Plant Biol. 2008;8(1):1-13.

37. Valdés-López O, Arenas-Huertero C, RamíRez M, Girard L, SÁNchez F, Vance CP, et al. Essential role of MYB transcription factor: PvPHR1 and microRNA: PvmiR399 in phosphorus-deficiency signalling in common bean roots. Plant Cell Environ. 2008;31(12):1834-43.

38. Barozai M, Din M, Baloch I. Structural and functional based identification of the bean (Phaseolus) microRNAs and their targets from expressed sequence tags. J Struct Funct Genomics. 2013;14(1):11-8.

39. Pelaez P, Trejo M, Iniguez L, Estrada-Navarrete G, Covarrubias A, Reyes J, et al. Identification and characterization of microRNAs in Phaseolus vulgaris by high-throughput sequencing. BMC Genomics. 2012;13(1):83.

40. Valdés-López O, Yang SS, Aparicio-Fabre R, Graham PH, Reyes JL, Vance CP et al. MicroRNA expression profile in common bean (Phaseolus vulgaris) under nutrient deficiency stresses and manganese toxicity. New Phytologist. 2010;187(3):805-18.

41. Kozomara A, Griffiths-Jones S. miRBase: annotating high confidence microRNAs using deep sequencing data. Nucleic Acids Res. 2014;42(D1):D68-73.

42. Zhang B, Pan X, Cannon CH, Cobb GP, Anderson TA. Conservation and divergence of plant microRNA genes. Plant J. 2006;46(2):243-59.

43. Schmittgen TD, Livak KJ. Analyzing real-time PCR data by the comparative C(T) method. Nat Protoc. 2008;3(6):1101-8

44. Hwang DG, Park JH, Lim JY, Kim D, Choi Y, Kim S, et al. The hot pepper (Capsicum annuum) microRNA transcriptome reveals novel and conserved targets: a foundation for understanding MicroRNA functional roles in hot pepper. PLoS One. 2013:8(5):e64238.

45. Khaldun AB, Huang W, Liao S, Lv H, Wang Y. Identification of MicroRNAs and Target Genes in the Fruit and Shoot Tip of Lycium chinense: A Traditional Chinese Medicinal Plant. PLoS One. 2015;10(1):e0116334.

46. Jones-Rhoades MW, Bartel DP, Bartel B. MicroRNAS and their regulatory roles in plants. Annu Rev Plant Biol. 2006;57:19-53.

47. Wu G, Poethig RS. Temporal regulation of shoot development in Arabidopsis thaliana by miR156 and its target SPL3. Development. 2006:133(18):3539-47.

48. Jung J-H, Seo P, Park C-M. MicroRNA biogenesis and function in higher plants. Plant Biotechnol Rep. 2009;3(2):111-26.

49. Nodine MD, Bartel DP. MicroRNAs prevent precocious gene expression and enable pattern formation during plant embryogenesis. Genes Dev. 2010;24(23):2678-92

50. Wu X-M, Liu M-Y, Ge X-X, Xu Q, Guo W-W. Stage and tissue-specific modulation of ten conserved miRNAs and their targets during somatic embryogenesis of Valencia sweet orange. Planta. 2011;233(3):495-505.

51. Yang L, Conway SR, Poethig RS. Vegetative phase change is mediated by a leaf-derived signal that represses the transcription of miR156. Development. 2011;138(2):245-9

52. Liu HH, Tian X, Li YJ, Wu CA, Zheng CC. Microarray-based analysis of stressregulated microRNAs in Arabidopsis thaliana. RNA (New York, NY). 2008;14(5):836-43.

53. Trindade I, Capitão C, Dalmay T, Fevereiro M, Santos D. miR398 and miR408 are up-regulated in response to water deficit in Medicago truncatula. Planta. 2010;231(3):705-16

54. Sunkar R, Li Y-F, Jagadeeswaran G. Functions of microRNAs in plant stress responses. Trends Plant Sci. 2012;17(4):196-203

55. Zhang B, Pan X, Cobb GP, Anderson TA. Plant microRNA: A small regulatory molecule with big impact. Dev Biol. 2006;289(1):3-16

56. Harborne JB: Phytochemistry of the Leguminosae. In: Phytochemical Dictionary of the Leguminosae. Edited by Bisby FA. London: Chapman and Hall; 1994.

57. Li YC, Korol AB, Fahima T, Beiles A, Nevo E. Microsatellites: genomic distribution, putative functions and mutational mechanisms: a review. Mol Ecol. 2002;11(12):2453-65.

58. Joy N, Asha S, Mallika V, Soniya EV. De novo Transcriptome Sequencing Reveals a Considerable Bias in the Incidence of Simple Sequence Repeats towards the Downstream of 'Pre-miRNAs' of Black Pepper. PLoS One. 2013;8(3):e56694.

59. La Rota M, Kantety R, Yu J-K, Sorrells M. Nonrandom distribution and frequencies of genomic and EST-derived microsatellite markers in rice, wheat, and barley. BMC Genomics. 2005:6(1):23.

60. Hisano H, Sato S, Isobe S, Sasamoto S, Wada T, Matsuno A, et al. Characterization of the soybean genome using EST-derived microsatellite markers. DNA Res. 2007;14(6):271-81.

61. Cloutier S, Niu Z, Datla R, Duguid S. Development and analysis of EST-SSRs for flax (Linum usitatissimum L.). Theor Appl Genet. 2009;119(1):53-63.

62. Akkaya MS, Bhagwat AA, Cregan PB. Length Polymorphisms of Simple Sequence Repeat DNA in Soybean. Genetics. 1992;132(4):1131-9.

63. Bell CJ, Ecker JR. Assignment of 30 microsatellite loci to the linkage map of Arabidopsis. Genomics. 1994;19(1):137-44.

64. Zhang Y. miRU: an automated plant miRNA target prediction server. Nucleic Acids Res. 2005;33(Web Server issue):W701-704.

65. Brodersen $P$, Sakvarelidze-Achard L, Bruun-Rasmussen M, Dunoyer $P$, Yamamoto YY, Sieburth L, et al. Widespread translational inhibition by plant miRNAs and siRNAs. Science (New York, NY). 2008;320(5880):1185-90.

66. Yu N, Cai W-J, Wang S, Shan C-M, Wang L-J, Chen X-Y. Temporal Control of Trichome Distribution by MicroRNA156-Targeted SPL Genes in Arabidopsis thaliana. Plant Cell. 2010;22(7):2322-35.

67. Goettel W, Liu Z, Xia J, Zhang W, Zhao PX, An Y-Q. Systems and Evolutionary Characterization of MicroRNAs and Their Underlying Regulatory Networks in Soybean Cotyledons. PLoS One. 2014;9(1):e86153.

68. Calviño M, Messing J. Discovery of MicroRNA169 Gene Copies in Genomes of Flowering Plants through Positional Information. Genome Biol Evol. 2013;5(2):402-17.

69. Sunkar R, Zhu J-K. Novel and Stress-Regulated MicroRNAs and Other Smal RNAs from Arabidopsis. Plant Cell. 2004;16(8):2001-19.

70. Zhou L, Liu Y, Liu Z, Kong D, Duan M, Luo L. Genome-wide identification and analysis of drought-responsive microRNAs in Oryza sativa. J Exp Bot. 2010;61(15):4157-68. 
71. Debernardi JM, Rodriguez RE, Mecchia MA, Palatnik JF. Functional Specialization of the Plant miR396 Regulatory Network through Distinct MicroRNA-Target Interactions. PLoS Genet. 2012;8(1):e1002419.

72. Abdel-Ghany SE, Pilon M. MicroRNA-mediated Systemic Down-regulation of Copper Protein Expression in Response to Low Copper Availability in Arabidopsis. J Biol Chem. 2008;283(23):15932-45.

73. Wan P, Wu J, Zhou Y, Xiao J, Feng J, Zhao W, et al. Computational Analysis of Drought Stress-Associated miRNAs and miRNA Co-Regulation Network in Physcomitrella patens. Genomics Proteomics Bioinformatics. 2011;9(1-2):37-44.

74. Griffiths-Jones S, Grocock RJ, van Dongen S, Bateman A, Enright AJ. miRBase: microRNA sequences, targets and gene nomenclature. Nucleic Acids Res. 2006;34 suppl 1:D140-4

75. Burge SW, Daub J, Eberhardt R, Tate J, Barquist L, Nawrocki EP, et al. Rfam 11.0: 10 years of RNA families. Nucleic Acids Res. 2013;41(D1):D226-32.

76. Benson DA, Karsch-Mizrachi I, Lipman DJ, Ostell J, Wheeler DL. GenBank. Nucleic Acids Res. 2005;33 suppl 1:D34-8.

77. Lamesch P, Berardini TZ, Li D, Swarbreck D, Wilks C, Sasidharan R, et al. The Arabidopsis Information Resource (TAIR): improved gene annotation and new tools. Nucleic Acids Res. 2012;40(Database issue):D1202-1210.

78. Schmutz J, Cannon SB, Schlueter J, Ma J, Mitros T, Nelson W, et al. Genome sequence of the palaeopolyploid soybean. Nature. 2010;463(7278):178-83.

79. Goodstein DM, Shu S, Howson R, Neupane R, Hayes RD, Fazo J, et al. Phytozome: a comparative platform for green plant genomics. Nucleic Acids Res. 2012:40(D1):D1178-86.

80. Hofacker IL, Fontana W, Stadler PF, Bonhoeffer LS, Tacker M, Schuster P. Fast folding and comparison of RNA secondary structures. Monatsh Chem. 1994;125(2):167-88

81. NG Kwang Loong S, Mishra SK. Unique folding of precursor microRNAs: Quantitative evidence and implications for de novo identification. RNA. 2007:13(2):170-87.

82. Schultes EA, Hraber PT, LaBean TH. Estimating the contributions of selection and self-organization in RNA secondary structure. J Mol Evol. 1999;49(1):76-83.

83. Huynen M, Gutell R, Konings D. Assessing the reliability of RNA folding using statistical mechanics. J Mol Biol. 1997;267(5):1104-12.

84. Moulton V, Zuker M, Steel M, Pointon R, Penny D. Metrics on RNA secondary structures. J Comput Biol. 2000;7(1-2):277-92.

85. Altschul SF, Gish W, Miller W, Myers EW, Lipman DJ. Basic local alignment search tool. J Mol Biol. 1990;215(3):403-10.

86. Zhang B, Pan X, Anderson TA. Identification of 188 conserved maize microRNAs and their targets. FEBS Lett. 2006;580(15):3753-62.

87. Martin M. Cutadapt removes adapter sequences from high-throughput sequencing reads. EMBnet J. 2011;17(1):10-12

88. Turner M, Adhikari S, Subramanian S: Optimizing stem-loop qPCR assays through multiplexed cDNA synthesis of U6 and miRNAs. Plant Signaling \& Behavior 2013;8(8):e24918.

89. Kramer MF. Stem-loop RT-qPCR for miRNAs. Current Protocols in Molecular Biology. 2001;95(15.10):1-15.

90. Dai $X$, Zhao PX: psRNATarget: a plant small RNA target analysis server. Nucleic Acids Res 2011, 39(Web Server issue):W155-159.

91. Kertesz M, lovino N, Unnerstall U, Gaul U, Segal E. The role of site accessibility in microRNA target recognition. Nat Genet. 2007;39(10):1278-84.

92. Barrett T, Wilhite SE, Ledoux P, Evangelista C, Kim IF, Tomashevsky M, et al. NCBI GEO: archive for functional genomics data sets-update. Nucleic Acids Res. 2013:41(D1):D991-5.

\section{Submit your next manuscript to BioMed Central and take full advantage of:}

- Convenient online submission

- Thorough peer review

- No space constraints or color figure charges

- Immediate publication on acceptance

- Inclusion in PubMed, CAS, Scopus and Google Scholar

- Research which is freely available for redistribution

Submit your manuscript at www.biomedcentral.com/submit 